\title{
Capitalismo y ética: una relación de tensiones
}

\section{Capitalism and ethics: a relationship of tensions}

\author{
DOI: https://doi.org/10.17981/econcuc.40.2.2019.02
}

Artículo de Revisión. Fecha de recepción: 28/01/2019. Fecha de aceptación: 18/03/2019

\author{
Carlos Alberto Gómez Cano \\ Universidad de la Amazonia (Florencia, Colombia) \\ car.gomez@udla.edu.co \\ Verenice Sánchez Castillo \\ Universidad de la Amazonia (Florencia, Colombia) \\ ve.sanchez@udla.edu.co \\ Edwin Eduardo Millán Rojas \\ Universidad de la Amazonia (Florencia, Colombia) \\ e.millan@udla.edu.co
}

Para citar este artículo:

Gómez, C., Sánchez, V. y Millán, E. (2019). Capitalismo y ética: una relación de tensiones. Económicas CUC, 40(2). 31-42. DOI: http:// dx.doi.org/10.17981/econcuc.40.2.2019.02

\section{Resumen}

La imponencia del sistema del modelo económico capitalista y su constante búsqueda por la multiplicación del capital ha permeado varios escenarios sociales, generando desequilibrios en las relaciones humanas, todo justificado en el quehacer económico. Sin embargo, la ética surge como un elemento de equilibrio y armonización de las tendencias salvajes de este modelo económico, la cual, busca consolidar un punto medio entre los intereses individuales y los colectivos. De esta manera, el presente artículo de corte cualitativo y revisión sistémica, buscó propiciar algunas consideraciones en torno al rol de la ética como generadora de equilibrio en el sistema capitalista, en el marco de una sociedad que demanda modelos más equitativos e incluyentes. Dentro de los hallazgos destaca que la transformación del capitalismo salvaje es posible solo a través de rigurosos procesos de educación, que permitan desvanecer el paradigma del egoísmo. Se concluye que la modificación del modelo capitalista requiere alineación de factores bastante complejos individualmente como son lo económico, político y cultural, lo cual implica largo tiempo para ser alcanzado.

Palabras clave: Ética; capitalismo; empresas; equilibrio; desarrollo integral

\begin{abstract}
The imposition of the capitalist economic model system and its constant search for the multiplication of capital has permeated various social scenarios, generating imbalances in human relations, all justified in economic activity. However, ethics emerges as an element of balance and harmonization of the wild tendencies of this economic model, which seeks to consolidate a midpoint between individual and collective interests. In this way, the present article of a qualitative nature and a systemic review, sought to promote some considerations regarding the role of ethics as a generator of balance in the capitalist system, within the framework of a society that demands more equitable and inclusive models. Among the findings highlights that the transformation of savage capitalism is possible only through rigorous processes of education, which allow the paradigm of selfishness to vanish. It is concluded that the modification of the capitalist model requires alignment of quite complex factors individually such as the economic, political and cultural aspects, which implies a long time to be reached.
\end{abstract}

Keywords: Ethics; capitalism; companies; balance; integral development 


\section{INTRODUCCIÓN}

\section{Empresas y capitalismo, aproximaciones}

Desde el momento mismo del abandono del modo de vida nómada, la creación y constitución de asentamientos y territorios forjó el desarrollo de dinámicas económicas en las nacientes comunidades (Gómez, Ramón y González, 2016). Entonces, como lo expresan Pérez, Hernández, Acosta y Chumaceiro (2009)

La conformación histórica y axiológica del Estado apunta hacía una concepción gregaria y de interés general, donde se dice que los hombres se agrupan por exigencias de supervivencia frente a su medio ambiente, se unen para organizar y distribuir el trabajo o para establecer un mecanismo de control frente a los intereses antagónicos, y donde se sobrepone un grupo dominante. Pero en el análisis de las implicaciones humanas el Estado se concibe de una u otra forma para alcanzar el bienestar general (p. 274).

En esta misma tónica, los autores Hernández, Chumaceiro y Ravina (2017) exponen que el "Estado es una estructura de poder institucional que refleja una dinámica entre los múltiples intereses que representa, es identificada con las élites de poder y en su función moderna da respuestas a acumulación y distribución de capital" (p. 50).

De tal manera, estas circunstancias han generado, a través de la evolución del tiempo y múltiples contextos sociales, la consolidación paulatina de varios sistemas económicos, los cuales han marcado diferentes etapas en la reciente historia de la humanidad, al punto de crear profundas diferencias entre países: guerras, estilos de liderazgo y múltiples concepciones de la vida en sociedad. En consecuencia, por razones que no son objeto de este artículo, el capitalismo se ha consolidado como el modelo económico pre- ponderante en el mundo, un contexto donde la posesión de capital y la construcción de riqueza han derrotado las barreras geográficas (Mateus y Brasset, 2002). En palabras de Moré (2014):

El capitalismo es un sistema regido por el mercado (oferta-demanda) a través del mecanismo de la competencia, por el cual cada individuo, buscando su mejoramiento propio, se enfrenta a una multitud de personas con motivaciones similares y tiene que adaptarse a los precios que ofrecen sus competidores ( $p$. 18).

La definición hace evidente dos elementos: competencia y mejoramiento. El sistema capitalista obliga a sus integrantes a estar en una constante lucha por la obtención de recursos, mismos que le permitirán generar mejores condiciones; nada distante de lo que considera la época primitiva, donde se luchaba por la mejor presa, la cual además de satisfacer sus necesidades, le permitía generar excedentes para tranzar (Santarcángelo y Borroni, 2012). Bajo esta lógica, sería imposible extraer a las empresas, quienes reflejan claramente el sistema capitalista, al existir un amplio espectro, desde las de tipo unipersonal hasta grandes plataformas industriales que cobijan millones de funcionarios.

Según el diccionario de la Real Academia Española-RAE (2014), las empresas son una "unidad de organización dedicada a actividades industriales, mercantiles o de prestación de servicios con fines lucrativos" (p. 1). En dicha definición se realiza especial énfasis en la generación de capital, asintiendo entonces que dentro de los objetivos primarios de las empresas -con algunas excepcionesestá la generación de utilidades económicas. En el caso de Colombia, los dirigentes políticos, quienes se encargan de los procesos de regulación, acogieron perfectamente este enfoque, pues el Decreto 410 (1971, art. 25) reza: 
Artículo 25. EMPRESA - CONCEPTO. Se entenderá por empresa toda actividad económica organizada para la producción, transformación, circulación, administración o custodia de bienes, o para la prestación de servicios. Dicha actividad se realizará a través de uno o más establecimientos de comercio.

De esta manera, es innegable como la mayoría de las empresas, especialmente aquellas que hacen parte del sistema capitalista, persiguen incasablemente un estatus de rentabilidad, sustentabilidad y competitividad (Robles, 2012). Esta posición es compartida por De Jaime (2010), quien afirma que el Objetivo Básico Financiero $(\mathrm{OBF})$ de las organizaciones gira en torno a la conseguir el mayor valor posible en el mercado, además, añade respecto al OBF que “(...) en la medida que la consecución de este objetivo garantiza la supervivencia y expansión de la empresa, se sitúa necesariamente por encima de otros objetivos funcionales por muy importantes que sean (...)" (p.20), es decir, se privilegia la generación de excedente por encima de otros objetivos, los cuales podrían estar relacionados con bienestar organizacional, sostenibilidad ambiental, entre otros.

\section{¿Cuál es el límite en la búsqueda de resultados económicos?}

Está pregunta tiene un muy alto grado de complejidad. Inimaginable para muchos, especialmente bajo la concepción de un sistema que privilegia la obtención de resultados por encima de todo (Barbosa, Medina y Vargas, 2014); parecería utópico entonces establecer un límite tratando de circunscribir al capitalismo, prácticamente sería una tarea antinatural de su filosofía. En palabras de Shenfield (1982):
El capitalismo ha sido atacado por muchas razones. Algunos lo acusan de ser un sistema ineficiente, inestable o autodestructivo en la producción de bienes y servicios. Otros dicen que es inmoral por naturaleza, puesto que premia, estimula o se sustenta en los impulsos inmorales del hombre en contra de sus tendencias morales, o que, a lo más, es moralmente neutro, contrario a las supuestas exigencias que requiere una sociedad bien constituida, cuyo sistema económico debe tener fuerza y carácter moral (p. 136).

La anterior disertación ratifica la ferocidad del capitalismo, la cual ha desbordado los límites tradicionales de la ética y la moral (Sabogal, 2015), dando paso a su instinto de generación de riqueza. Sin embargo, el asunto puede encontrar su punto de partida en la génesis misma de la empresa (unidad de negocios), para ello, conviene retomar lo planteado por Dávalos (2010), quien expone que:

“(...) la empresa es la actividad que le da carácter de comerciante a una persona. La empresa es una actividad que consiste en coordinar y organizar tanto elementos corpóreos como incorpóreos, así como el trabajo de las personas para producir e intercambiar bienes o servicios (...)" (p. 101).

En la precitada definición aflora un elemento muy importante, el cual puede establecer límites al desaforado instinto salvaje del capitalismo, la condición humana. Es innegable, pese a la filosofía y dinámica de cualquier modelo económico, que son los humanos quienes lo operan, desarrollan y posicionan, de ahí, por robusto y eficiente que sea, siempre existe la posibilidad de ajustar y reinventarse, según la condición e intención de sus operadores. Bajo esta lógica, será la condición humana la única fuerza opositora al capitalismo salvaje, pues puede anteponer condiciones generales por 
encima de la dinámica del modelo, siendo la única ventana para un capitalismo regulado y equilibrado.

En tal sentido, el presente artículo, fruto de un ejercicio de revisión documental, tendrá como objetivo resaltar la importancia de la ética en el capitalismo, como una ventana para la construcción de una sociedad, que debe estar abierta al cambio, la competencia y una constante lucha por el crecimiento, manteniendo equilibrios dentro de su dinámica económica, estableciendo límites y barreras a la competencia salvaje de la generación de capital, a fin de preservar, aunque sea en mínimas proporciones, el componente humano y social de nuestra sociedad.

\section{Metodología}

El derrotero metodológico del manuscrito estuvo orientado bajo un enfoque cualitativo, implementando un método documental y descriptivo según el protocolo de Gómez, Sánchez y Jiménez (2016). El componente documental se evidencia en la pesquisa realizada en leyes, tesis, artículos y libros; por su parte, la clasificación, triangulación y conformación de las categorías de análisis configuran la tendencia descriptiva (Merino, 2011). Respecto al método, se surtió de la siguiente manera:

- Etapa exploratoria: Se consultaron diferentes fuentes relacionadas con el objeto planteado en el escrito, donde se lograron establecer posiciones en torno al tema en cuestión, situación que generó discusión académica.

- Etapa de organización y sistematización: Con los resultados de la etapa anterior, se estableció una matriz de análisis simple, donde se clasificó la información por componentes de análisis, permitiendo establecer los acápites del manuscrito.
- Etapa de resultados y conclusiones: Finalmente, en esta etapa de generó un hilo conductor entre los apartados definidos, y además, se construyeron las reflexiones finales, las cuales se consignaron en el capítulo de conclusiones.

El propósito de este tipo de manuscritos, según indica Sánchez (2011), es sintetizar conocimientos fragmentados, así como la contribución a la generación de discusiones académicas, mismas dan paso a nuevos procesos de apropiación social y académica del saber (Sánchez, 2016).

\section{RESUltAdOS Y DISCUSIÓN}

\section{La ética en el sistema capitalista: una relación de tensiones}

Los conceptos de ética en la actual sociedad del conocimiento son abundantes y diversos, pues se han abordado desde diferentes aristas, lo que representa un sinfín de posiciones en torno al tema, en palabras de Sisiruca y Salazar (2014) y Hernández, Chumaceiro, Zirit y Acurero (2018), el dilema ético parte de los valores morales relacionados con el hombre y su modo de actuar ante determinada situación, son adquiridos desde el seno de la familia. Sin embargo, para el caso que nos convoca, la reflexión se orientará por lo planteado por Betancur (2010), quien expresa:

[...] se entiende por ética la reflexión que se realiza sobre los actos humanos, en cuanto ellos comportan, de manera individual o colectiva, una orientación hacia la práctica del bien, o hacia la exclusión de éste que se entiende como la práctica del mal. Lo correcto (bien) y lo incorrecto (mal) se entenderán con referencia a la dignificación de los seres humanos y el cumplimiento de unos mínimos universales que permiten hacer juicios de valor acerca de las consecuencias que tienen las acciones personales y colectivas (corporativas) (p. 77). 
En línea con lo anterior, tal y como se citó en la introducción, la ética aparece como un elemento con la capacidad de apaciguar y equilibrar el instinto salvaje del capitalismo (Román, 2011). Sin embargo, es una relación sensible, donde el sujeto (empresario) está constantemente sometido a dilemas, generando entonces una relación tensionante entre estos dos agentes, pues, tal y como lo detalla Nikonov (2012): "basar el intercambio de mercado en el principio de igualdad anula la razón fundamental del intercambio: mejorar la situación de las partes que lo realizan" (p. 103).

Adicionalmente, el precitado autor expone que los temas éticos y morales dentro del sistema capitalista son asuntos extremadamente complejos, pues desvirtúan la esencia misma de su génesis, al punto de considerarse antinaturales en el sistema (Vinolo, 2012). Para Noguera (2007), el sistema capitalista genera motivaciones egoístas e insolidarias en los individuos, excluyendo posibilidades altruistas y comunitarias, esta situación es ratificada por Zorro (2009), al expresar:

Dentro de la lógica del capitalismo imperante en los países occidentales y que tiende a extenderse a la mayor parte del mundo, las decisiones económicas se han sustentado en una ética individualista, de carácter utilitarista, en la que el rendimiento económico es el criterio fundamental para las decisiones y en que la idea de justicia queda reducida al cumplimiento de acuerdos entre agentes económicos, independientemente de las condiciones de cada uno y del contexto que los rodee (p. 271).

No obstante, existen posiciones que dan a la ética un papel un poco más representativo dentro del sistema capitalista, donde le apuestan a su rol de equilibro entre los beneficios económicos perseguidos y el bienestar general. Por ejemplo, Giraldo (2007), sostiene que "la ética de los negocios parece ser entonces la que permite fundar el alma salvadora ante las desmedidas de la economía moderna" (p. 60). Según Lalama y Bravo (2017), base del capitalismo social, con el cual se pretende humanizar el sistema; escenario donde las empresas sean respetuosas de los recursos naturales (sostenibilidad) y la sociedad.

De esta manera, la ética es vista como una alternativa para la transformación de las dinámicas sociales y empresariales (Agudelo, 2013); y para algunos casos específicos su rol en el capitalismo, según Méndez (2005), el cambio no radica en la eliminación de los objetivos económicos, pues ellos desvanecerían la esencia del modelo, sino, en añadir los elementos y variables en el desarrollo misional de la organización, es decir, contemplar otro tipo de dimensiones, las cuales, generalmente, están ligadas al beneficio de las comunidades en el entorno de la empresa, especialmente en factores sociales y medioambientales (Trujillo y Vélez, 2006), es decir, re-significar la ética social y por ende la del capitalismo (Aliga, 2010).

En concordancia, al referirnos al componente ético dentro del capitalismo no se busca eliminar el modelo, sino adaptarlo y ajustarlo, para que, sin desconocer los intereses de los dueños del capital, exista un instante para la reflexión conjunta (Mariani, 2008), donde la sociedad también tenga dividendos, no necesariamente económicos, pero sí que redunde en la calidad de vida de sus habitantes (Giavedoni, 2015), es un asunto complejo y que demanda la transformación y erradicación de muchos paradigmas en la sociedad, pero que al final debe ser visto como una relación de gana-gana, donde existe un beneficio para todos, lejos de los sometimientos, abusos, desmanes y excesos propios del salvajismo económico. En palabras de Berzosa (2013): 
La ética deber servir para denunciar situaciones abusivas, como pueden ser la existencia de la pobreza y del hambre, largas jornadas de trabajo, la sobreexplotación del trabajo de mujeres y niños, las condiciones lamentables en las que se trabaja, la falta de derechos laborales y sindicales, la dominación que ejercen los países ricos sobre los pobres, entre otras cosas" (p. 275).

La anterior afirmación deja en evidencia el papel de la ética como un elemento inherente a la conciencia de los dirigentes de grandes capitales, pues no critica la búsqueda de riqueza, siempre y cuando se respeten las condiciones humanas, rechazando entonces cualquier situación de sometimiento y represión. Es ahí, precisamente en la delgada línea que separa estos dos grandes intereses, donde se puede afirmar que la ética, en un sistema como el capitalista, busca el respeto y la dignificación de las acciones humanas, sin perjuicio del cumplimiento de los fines y propósitos de las empresas.

\section{Economía Solidaria:}

un creciente hito de resistencia

contra el Capitalismo Salvaje

Si bien el capitalismo salvaje se ha consolidado como el modelo prevalente para el desarrollo de la economía mundial, fruto de algunas crisis, conflictos y cuestionamientos en torno a sus prácticas y efectos sobre la sociedad, se han generado rutas alternas al interior del mismo sistema (Dabat, Hernández y Vega, 2015), las cuales privilegian en mayor medida el componente humano, mostrando un lado más sostenible del sistema, "es precisamente la satisfacción de necesidades del ciudadano en el campo de su patrimonio, su familia y la institucionalidad" (Zuñiga, 2017, p. 189). Una de dichas iniciativas es la Economía Solidaria, la cual, según Obando (2009), se puede definir como el "conjunto heterogéneo de concepciones y enfoques teóricos, realidades socioeconómicas e institucionales y prácticas empresariales y asociativas que desde el último cuarto del $\mathrm{XX}$, vienen desarrollando un creciente sentido de pertenencia a una forma diferente de entender el papel de la economía y los procesos económicos en las sociedades contemporáneas" (p. 92).

Aunado a lo anterior, Chumaceiro, Hernández y Chirinos (2016) expresan:

(...) en las últimas décadas, la atención dirigida hacia el medio ambiente ha dejado de ser patrimonio específico de la ecología para dar lugar a una amplísima y variada literatura dentro de las ciencias sociales: en el derecho, la geografía, la economía, la antropología, la sociología y la ciencia política (p. 56).

Dichas prácticas, en el campo específico de la economía, afirma Díaz (2015), consiste en "una forma de producir, intercambiar, consumir y distribuir la riqueza, centrada en la valorización del ser humano - $-\mathrm{y}$ no en el capital—, que tiene como base la asociatividad y la cooperación, de tipo autogestionaria, con la finalidad de asegurar la reproducción ampliada de la vida" (p. 44). "En otras palabras, las experiencias de economía social no sólo producen bienes, valores de uso, sino que producen sociedad, producen relaciones sociales" (Giavedoni, 2015, p. 197). Sin embargo, esta tarea es altamente compleja, pues, tal y como lo afirma, Márquez (2010), el capitalismo salvaje no se restringe en "desmantelar la economía de subsistencia, cancelar o menguar los apoyos a la economía social" (p. 442), deteriorando la calidad de vida y del trabajo de la población, situación antinatural a la génesis de la economía solidaria (Vélez e Insuasty, 2017). 
Aunado a lo anterior, la Organización de las Naciones Unidas - ONU (2014), expresa que la economía solidaria es una aliada estratégica para el cumplimiento de los objetivos de desarrollo sostenible, pues las recientes crisis sociales, los incontrolables indicadores de pobreza y aumento de la desigualdad, demandan al sistema económico la generación de alternativas que favorezcan los desarrollos sociales y con esto las metas establecidas en el mediano y largo plazo en los diferentes planes, agendas y apuestas a nivel mundial (Evers y Laville, 2004).

Lo anterior es ratificado por Parente (2014), quien afirma que la economía solidaria tiene la "capacidad de generar ocupación e ingreso para aquellas personas excluidas de la sociedad y que no tendrían otra forma de inserción en el mercado de trabajo" (p. 176). Este tipo de iniciativas, según Feijó, Feijó y Ormaza (2014), permiten "la construcción de actores colectivos y redes socioeconómicas insertas en entramados productivos y cadenas de valor locales y regionales, en donde la persona es y será lo central y donde los valores, los derechos, la dignidad y la confianza sean los motores y el fin de una sociedad para el buen vivir" (p. 8). Esta dinámica se convierte en un hito de cambio y transformación, despojando al capital como el epicentro de las acciones organizaciones. En resumidas cuentas, se cumple lo expresado por Álvarez (2017), quien afirma que:

En conclusión, la economía social y solidaria resurge como opción organizacional para atender las nuevas tendencias de consumo responsable, producción sostenible, comercio justo, finanzas éticas y otro sinfín de iniciativas que siguen un camino distinto al de la mercantilización, extractivismo y explotación propio de los actuales momentos y que constituye un sistema económico insostenible y un paradigma en crisis (p. 15).
Del egoísmo al pensamiento colectivo: un desafio de la ética al capitalismo, fundamentado en la acción transformadora de la educación

Luego de las disertaciones realizadas en los apartados anteriores, es posible afirmar como la batalla por librar la ética en el capitalismo está relacionada directamente con el egoísmo que se cimienta en la obtención de beneficios individuales, los cuales se convierten en una bandera de éxito personal, sin importar que estos disten profundamente de los intereses colectivos y del bien común (Pérez, 2010). Sobre el particular, Leriche, Sosa y Coloca (2009), expresan dentro de las conclusiones de su estudio que:

(...) se observa que el individuo representativo de la economía positiva no es empático, lo cual resulta deficiente en el momento en que éste tendría que buscar el bienestar de los demás. El egoísmo es una cuestión aún más cuestionable, puesto que el que el individuo sea egoísta y persiga su propio interés detenta el hecho de que no es altruista y por tanto, no busca cooperar con los demás, puesto que sólo vela por sí y no considera los intereses de los demás (...) (p. 115).

Producto de la reflexión de la anterior definición es innegable que la naturaleza del capitalismo, apoyada en la tendencia individualista de los seres humanos, se ha propagado como un método de gestión y desarrollo (Álvarez, 2016). No obstante, la ética, como se mencionó con anterioridad, puede ser la contrapartida de esta tendencia, pero ¿En qué momento realiza su intervención protagónica?, pues, a diferencia de algunos economistas que comparten plenamente los planteamientos de Adam Smith acerca de una mano invisible como agente regulador del mercado, no existe rastro alguno de una conciencia invisible, la cual tenga como función regular éticamente los conflictos (Vinolo, 2012). 
En tal sentido, se hace imperioso vincular una nueva variable a la discusión, la educación. La cual, siguiendo los planteamientos de Sánchez, Gómez y Ramón (2016), es una fuerza transformadora, liberadora y capaz de reconfigurar varios escenarios sociales, dentro de los cuales, por supuesto, se pueden incluir el egoísmo que acompaña al modelo capitalista tradicional. Sin embargo, esta iniciativa demanda de una construcción colectiva, paulatina y sólida, donde solo se podrán obtener resultados en el largo plazo, luego de consolidar toda una estructura social que propenda por el equilibrio en cuestión (Mendy y Gallo, 2013).

Esta posición es compartida por Castellanos (2015), al afirmar que la educación de los ciudadanos en valores y ética es una estrategia para salvaguardar la seguridad pública, pues genera en el individuo una serie de cuestionamiento que, según sus conclusiones, permiten disminuir la posibilidad de cometer hechos que atenten contra el bien común. Este escenario genera un desafío para los educadores, pues como lo afirma Díaz, Montaner y Prieto (2007):

La ética no se decreta, es una forma de vida, (...) por lo tanto si en cada documento, cada decisión, cada discurso, cada clase, cada evaluación, no se le da y se le exige actitudes éticas a los alumnos orientadas a un pensamiento colectivo ganar-ganar, donde ellos perciban como actitudes preferibles aquellas que tienen que ver con el bienestar común, no se puede pretender que cuando salgan al mercado de trabajo se conviertan en tomadores de decisiones orientadas a la equidad y la justicia social (...) (p. 169).

De esta manera, siguiendo a Cota (2002), la educación en valores tiene un gran impacto en la significación y transcendencia de los desarrollos sociales, convirtiéndose en una base fundamental para el progreso de los pueblos, de ahí que, la sociedad, a través de la educación, debe generar altos componentes éticos en los dirigentes capitalistas, a fin de que reconozcan y nivelen la importancia del bien común sobre sus intereses individuales, y, tal y como se ha mencionado con anterioridad, se obtengan sus resultados sin desconocer a la sociedad, ratificando entonces la relación gana-gana.

En definitiva, utilizando a la educación como herramienta estratégica para la difusión de la ética entre los actuales y futuros dirigentes del sistema capitalista (Tueros, 2006), se busca consolidar una sociedad equilibrada, regulada y consciente, que aun en los tiempos de competencia y lucha por la generación de excedentes financieros, se deben considerar factores humanos y sociales, además, de respetar los otros agentes propios del desarrollo sostenible de los pueblos. Esta situación, aunque pareciera ser una quimera, puede ser una realidad si confluyen los intereses del Estado, la ciudadanía y, por supuesto, de los señores capitalistas. La transformación del capitalismo salvaje es posible solo a través de rigurosos procesos de educación, que permitan desvanecer el paradigma del egoísmo.

\section{Conclusiones}

La fuerza del capitalismo como modelo económico es innegable, por ello, aunque no es una idea descabellada, considerar su erradicación - o trasformación - para dar paso a un nuevo sistema es una tarea de grandes dimensiones, que exigirá la alineación de una serie de factores de tipo económico, político y cultural bastante complejos, siendo entonces una tarea de largo aliento. Sin embargo, mientras ocurre un evento con la fuerza suficiente para motivar esa transformación, se hace imperioso humanizar las tendencias capitalistas, es decir, apoyar la acumulación de capital, la competencia y la 
lucha por el podio en el mercado, pero sin dejar atrás los intereses comunes, el respeto por los demás individuos y nuestro entorno.

En tal sentido, la ética, como producto de una construcción social que propende por la consolidación de una actuar ideal, surge como un elemento capaz de neutralizar la filosofía del capitalismo salvaje, dando paso, aunque sea en pequeñas proporciones, a cuestionamientos internos que redunden en el pensamiento colectivo, dejando, por algunos instantes, el individualismo y egoísmo propios de un modelo que superpone los beneficios individuales sobre todo contexto. Es decir, la ética puede moldear y limitar la fuerza voraz del capitalismo, generando un equilibrio entre las fuerzas individuales y colectivas.

Dicha tarea, aunque en principio parece titánica, ya tiene algunos avances como la economía solidaria, donde, pese a existir la búsqueda de recursos y beneficios económicos, el epicentro de las organizaciones migra del paradigma de la utilidad al bienestar social, donde mas importante que los excedentes financieros son las condiciones de vida óptima de todos los integrantes de la sociedad. Estas iniciativas parecieran validar las teorías relacionadas con el que capitalismo es tan solo una forma económica, y que su salvajismo está en quienes lo desarrollan, es decir, la condición egoísta del ser humano.

Ahora bien, los dos anteriores escenarios serán posibles, si y solo si, la educación interviene de forma decidida, pues su solo a través de su rol transformador se podrán generar elementos en la consciencia de todos los individuos, amén de que se sientan parte de un todo, el cual está compuesto por otras personas y no solo por su ego triunfador que se satisface a través de la generación de utilidades, sin importar el costo de las mismas. La educación se convierte en el superhéroe de la historia, una especie de agente generador de equilibrio entre la génesis del modelo y el ideal de sociedad justa y equitativa.

\section{REFERENCIAS}

Agudelo, A. (2013). Aporte ético de la familia y el sistema educativo a las organizaciones. Horizontes Pedagógicos, 15 (1),104-116.

Aliga, F. (2010). Ética y capitalismo en el siglo XXI. Intersticios, 4(1), 253-256

Álvarez, J. (2017). Economía social y solidaria en el territorio: significantes $y$ co-construcción de políticas públicas. Bogotá, D.C.: Fundación Cultural Javeriana de Artes Gráficas

Álvarez, E. (2016). Individualismo e identidad humana. Valenciana, (3), 37-50.

Barbosa, D., Medina, C. \& Vargas, M. (2014). Globalización, capitalismo financiero y responsabilidad social empresarial: tensiones estructurales. $\mathrm{Ci}$ vilizar 14(27), 135-154.

Berzosa, C. (2013). La difícil relación entre ética y economía. Revista de Economía Mundial, (35), 271-284

Betancur, J. (2010). La ética de la responsabilidad social empresarial. Katharsis, (9), 73-91

Castellanos, M. (2015). La ética en la sociedad actual y la importancia de la formación en valores durante la educación universitaria. Reconstitución de Instituciones, 1(3), 30-39.

Chumaceiro, A., Hernández, J. y Chirinos, E. (2016). Responsabilidad social universitaria, desarrollo sostenible y ciudadanía ambiental. Cuadernos de $R S O, 4(1), 53-64$

Cota, A. (2002). La importancia de los valores en el desarrollo humano de la organización. [Tesis Magistral]. Universidad Autónoma de Nuevo León, Nuevo León, México. 
Dabat, A., Hernández, J., Vega, C. (2015). Capitalismo actual, crisis y cambio geopolítico global. Economía UNAM, 12(36), 62-89. https://doi. org/10.1016/j.eunam.2015.10.005

Dávalos, M. (2010). Manual de introducción al derecho mercantil (1 ed.). México, D.F.: Nostra Ediciones S.A.

De Jaime, J. (2010). Las claves del análisis económico-financiero de la empresa (2 ed.) Madrid: ESID Editorial.

Díaz, J. (2015). Economías Solidarias en América Latina. México, D.F.: ITESO

Díaz, J., Montaner, J. \& Prieto, M. (2007). Formación ética para un desarrollo sustentable. Revista de Ciencias Sociales, 13(1), 159-171.

Evers, A. \& Laville, J. L. (2004). Defining the third sector in Europe. In E. Elgar, The third sector in Europe. UK: Elgaronline. Available from: https://www.elgaronline.com/ view/9781843764007.xml

Feijó, N., Feijó, E. y Ormaza, M. (2014). Economía Solidaria: un enfoque social hacia el Desarrollo Local. ECA Sinergia, 5(1), 1-8.

Giavedoni, J. (2015). Economía social y solidaria trabajo y capitalismo. Relación entre forma de trabajo y patrón de acumulación en el gobierno de la fuerza de trabajo. Trabajo y Sociedad, 25, 193-213.

Giraldo, G. (2007). Racionalidad y ética en las organizaciones. Cuadernos de Administración, (38), 53-62

Gómez, C., Ramón, L. y González, G. (2016). Reflexiones en torno a las políticas públicas. Faccea, 6(1), 12-20.

Gómez, C., Sánchez, V. y Jiménez, E. (2016). Factores endógenos relacionados con la permanencia irregular en las IES, una aproximación conceptual. En-Contexto, 4(5), 285-306.
Hernández, J., Chumaceiro, A. y Ravina, R. (2017). Estado populista y gestión de políticas sociales. Una mirada en América Latina. Negotium, 38(13), 4961

Hernández, J., Chumaceiro, A., Ziritt, G. y Acurero, M. (2018). Cultura para la paz en Colombia. Una aproximación desde las políticas públicas. Opción, 34(86), 612-641

Lalama, R. \& Bravo, A. (2017). Capitalismo social: un vistazo a resultados macroeconómicos de Ecuador, Perú y Colombia. Retos, 7(13), 91-104.

Leriche, C., Sosa, V., y Caloca, O. (2009). Economía y ética. Una revisión con base en la teoría del bienestar. Polis, 8(23), 95-118.

Mariani, R. (2008). Democracia, Estado y construcción del sujeto (ciudadanía). En, Programa de las Naciones Unidas para el Desarrollo, Democracia /Estado /Ciudadania: Hacia un Estado de y para la Democracia en América Latina (pp. 79-98). Lima: PNUD.

Márquez, H. (2010). Crisis del sistema capitalista mundial: paradojas y respuestas. Polis, 9(27), 435-461. http://dx.doi.org/10.4067/ S0718-65682010000300020

Mateus, J. y Brasset, D. (2002). La globalización: sus efectos y bondades. Economía y Desarrollo, 1(1), 65-77

Méndez, M. (2005). Ética y responsabilidad social corporativa. Ética y Economía, (823), 141-150

Mendy, G. \& Gallo, M. (2013). Educación transformadora: una propuesta universitaria de inclusión educativa y articulación territorial en economía social y solidaria. En, UNPL, Argentina en el escenario latinoamericano actual: debates desde las ciencias sociales, VII Jornadas de Sociología, Universidad Nacional de La Plata, La Plata, Argentina. 
Merino, A. (2011). Como escribir documentos científicos (Parte 3). Salud en Tabasco, 17(1-2), 36-40.

Moré, E. (2014). Sistemas económicos y modelos de economía moderna. Bogotá. D.C.: Editorial Universidad Autónoma de Colombia.

Nikonov, L. (2012). La lógica moral de la igualdad y la desigualdad en la sociedad de mercado. En, Fundación para el Progreso (Coord.). La moralidad del capitalismo (pp. 103-114). Santiago de Chile: Fundación para el Progreso.

Noguera, J. (2007). Capitalismo y justicia: los términos de la cuestión. Sistema 200, 87-106

Obando, D. (2009). Economía solidaria: ¿en función de un desarrollo alternativo o de un neocapitalismo? Alteridad 4(1), 88-97.

ONU. (2014). La Economía Social y Solidaria y el Reto del Desarrollo Sostenible. Ginebra: TFSSE

Parente, S. (2014). Economía solidaria, desarrollo local y microfinanzas: una convergencia posible. [Tesis Doctoral]. Universidad Autónoma de Madrid, Madrid, España.

Pérez, M. E., Hernández, J., Acosta, I., Chumaceiro, A. (2009). Consideraciones teóricas para el análisis de las Pequeñas y Medianas Empresas como fuente de generación de empleo y su correspondencia ética con la Sociedad. Revista Formación Gerencial, 8(2), 272-297

Pérez, R. (2010). El comportamiento moral en las organizaciones: una perspectiva desde la ética de la empresa. [Tesis Doctoral]. Universidad Complutense de Madrid, Madrid (España).

RAE. (2014). Concepto de Empresa. [Online]. Recuperado de http://dle.rae. es/?id=EsuT8Fg
República de Colombia. Presidencia de la Republica. (marzo 27 de 1971). Artículo 25. Por el cual se expide el Código del Comercio. [Decreto 410]. DO: 33.339

Robles, C. (2012). Fundamentos de administración financiera. México, D.F.: Red Tercer Milenio S.C.

Román, A. (2011). La huella de San Agustín en la ética de los valores de Scheler. [Tesis de Doctoral]. Universidad de Murcia, Murcia, España.

Sabogal, J. (2015). El modo de producción capitalista, su actual crisis sistémica y una alternativa posible. Sociedad $y$ Economía, 28, 75-94.

Sánchez, A. (2016). El género artículo científico: escritura y análisis desde la alfabetización académica y la retorica funcional. Medellín: Católica del Norte Fundación Universitaria.

Sánchez, A. (2011). Manual de redacción académica e investigativa: cómo escribir, evaluar y publicar artículos. Medellín: Católica del Norte Fundación Universitaria

Sánchez, V., Gómez, C. y Ramón, L. (2016). La Educación Superior en Colombia: una cuestión de calidad, no de cantidad. Criterios, 23(1), 141-168

Santarcángelo, J. y Borroni, C. (2012). El concepto de excedente en la teoría marxista: debates, rupturas y perspectivas. Cuadernos de Economía, 31(56), 1-20.

Shenfield, A. (1982). El capitalismo examinado por la ética. Estudios Públicos, 6, 135-152.

Sisiruca, M. \& Salazar, C. (2014). Valores éticos de la responsabilidad social interna en centros de producción audiovisual. Económicas CUC, 35(1), 79-90. Recuperado a partir de https://revistascientificas.cuc.edu.co/economicascuc/article/view/259 
Trujillo, M. y Vélez, R. (2006). Responsabilidad ambiental como estrategia para la perdurabilidad empresarial. Universidad \& Empresa, 5(10), 291-308.

Tueros, E. (2006). El educador, sujeto ético y político. Educación, 15(29), 35-51.

Vélez, A. e Insuasty, A. (2017). Experiencias de economía solidaria en escenarios de postconflicto. Medellín: Grupo de Investigación y Editorial Kavilando.

Vinolo, S. (2012). A qué le llaman moralizar el Capitalismo. Caminando con Adam Smith. Analecta política, 2(3), 37-53

Zorro, C. (2009). Ética, justicia e ideología en el desarrollo. Polis, 8(23), 247-278

Zuñiga, J. (2017). De la función económica del cheque, del cheque común al de pago diferido. Jurídicas CUC, 13(1), 183-198. https://doi.org/10.17981/juridcuc.13.1.2017.08

\section{Biodata}

Carlos Alberto Gómez Cano es Contador Público de la Universidad de la Amazonia (Colombia); Administrador Público, Escuela Superior de Administración Pública - ESAP (Colombia); Especialista en Pedagogía, Universidad de la Amazonia; Especialista en Gestión Pública, Escuela Superior de Administración Pública - ESAP; Magister en Ciencias de la Educación, Universidad de la Amazonia; Magister en Gestión y Evaluación de Proyectos de Inversión, Universidad Externado (Colombia). Docente-Investigador de la Universidad de la Amazonia y líder del Colectivo de Investigación en Educación, Naturaleza, Cultura e Innovación para la Amazonia - CIENCIA. https://orcid.org/0000-0003-0425-7201

Verenice Sánchez Castillo es Ingeniera Agroecóloga de la Universidad de la Amazonia; Magister en Estudios Regionales en Medio Ambiente y Desarrollo, Universidad Iberoamericana de Puebla (México); Doctora en Antropología, Universidad del Cauca (Colombia). Docente-Investigadora de la Universidad de la Amazonia y líder del Grupo de Investigación en Agroecología y Desarrollo Rural - GIADER. https://orcid. org/0000-0002-3669-312

Edwin Eduardo Millán Rojas es Ingeniero de Sistemas de la Universidad Distrital Francisco José de Caldas (Colombia); Especialista en Ingeniería de Software, Universidad INCCA (Colombia); Magíster en Ciencias de la Información y las Comunicaciones de la Universidad Distrital Francisco José de Caldas (colombia); Doctor en Ingeniería con énfasis en Ciencias de la Información y el Conocimiento, Universidad Distrital Francisco José de Caldas. Docente-Investigador de la Universidad de la Amazonia y líder del Grupo de Investigación en Informática, Innovación y Tecnología de la Universidad de la Amazonia (GITUA). https:// orcid.org/0000-0002-4258-4601 